\title{
Experimentelle Untersuchungen über Entstehung von Astigmatismus durch nicht-perforirende Corneawunden.
}

\author{
Von \\ Dr. L. J. Lans \\ in Leiden.
}

Mit 26 Figuren im Text.

Aus dem Laboratorium von Prof. W. Koster Gzn. in Leiden.

Es ist bekannt, dass man bei Astigmatikern nicht selten durch Cylindergläser keine Besserung erzielt. So haben wir für den irregulären Astigmatismus bis jetzt keine Gläser, welche die Brechung in allen Meridianen ungefähr gleich machen; ebenso wenig fiir Fälle, in welchen die Achsen der schwächsten und der stärksten Brechung nicht senkrecht aufeinander stehen.

Aber auch wenn die Correction gelingt, ergiebt sich noch eine Reihe von Schwierigkeiten. Ausser den gleichen Beschwerden wie bei Correction durch sphärische Gläser tritt bei Astigmatikern eine besondere Schwierigkeit auf, die bei Anwendung von gleich starken sphärischen Gläsern fehlt. Denn sobald die Gläser nicht genau centrirt sitzen, treten undeutliche Bilder auf. Dieser Anforderung nachzukommen, bereitet den Patienten häufig Unbequemlichkeiten; denn sobald die Augen von der Mittelstellung abweichen, ist die oben geforderte Voraussetzung nicht mehr gegeben. 
Wenn der Patient ein nach links oder nach rechts von $\mathrm{ihm}$ gelegenes Object ansehen will, so muss er den ganzen Kopf nach links oder nach rechts drehen, bis das Object sich in der Medianebene befindet. Will er nach oben oder unten seh c, so kann er sich ebensowenig seiner Augenmuskeln bediene, falls er scharf sehen will. Ausserdem muss er den Kopf "i verticaler Stellung halten, denn bei Senkung vor- oder rü 'wärts machen die Augen eine symmetrische Rollbewegung , m die Gesichtsachse, wodurch die Achse des Cylinderglases $u$ d der Meridian von stärkster; respective schwächster Brechung icht mehr zusammen fallen.

Ebenso machen sich Neigun, en des Kopfes nach der Seite unangenehm bemerkbar; denn ، "ch hierbei nimmt durch die parallele Rollbewegung das Augi hinter dem Glas eine veränderte Stellung ein, bei welcher der Astigmatismus nicht mehr vollständig corrigirt ist. Nicht selten klagen Astigmatiker, dass sie auf dem Sopha liegend, oder wenn sie den Kopf auf die Hand stützen, nicht oder doch nur mühsam lesen können. Wie bekannt, hat diese Beobachtung Javal zur Entdeckung der parallelen Rollbewegung der Angen geführt.

Es ist daher wohl berechtigt, nach einem anderen Nittel zur Correction des As. zu suchen.

Nun ist es eine altbekannte und von Fall zu Fall sich immer wieder ergebende Thatsache, dass nach ausgedehnten perforirenden Cornea-Wunden Astigmatismus auftritt. Sie legt den Versuch nahe, Astigmatismus künstlich zu erzeugen, und einen bestehenden zu ändern, respective auszugleichen. Die nächste Aufgabe ist nun, die Gesetze von Art und Richtung der Krümmungsänderung kennen zu lernen.

Natürlich ist die erste Bedingung bei jedem operativen Eingriff, dass die angewandte Methode keine Gefahr für Erhaltung des Auges oder des Sehvermögens mit sich bringt; denn in diesem Fall würde der Brille der Vorzug gehïhren. 
Experiment. Untersuch. äber Entstehung von Astigmatismus etc. 119

Bemerkenswerth ist, dass fast alle, die bisher eine Heilung auf operativem Wege erstrebt haben, perforirende Wunden der Cornea gemacht haben; eine solche Operation ist aber immerhin nicht ganz gefahrlos. Weiter ist auffallend, dass sie diese Incision immer nur auf einer Seite am Limbus corneae angebracht haben. Man sollte doch denken, dass diese Methode geeignet wäre, irregulären As. zu erzeugen. Wir wollen die Resultate dieser Versuche hier kurz zusammenstellen; denn sie sind von Belang für das Studium der nicht perforirenden Wunden, welches die Aufgabe dieser Arbeit ist.

Snellen hat sich schon lange für die Möglichkeit ausgesprochen, den Astigmatismus auf operativem Wege zu heilen; so legt er auch in geeigneten Fällen bei der Extraction dem Schnitt entsprechend den Meridian der schwächsten Krümmung an.

Bates ${ }^{1}$ ), Faber ${ }^{2}$ ), Schiötz ${ }^{3}$ ), Lucciola ${ }^{4}$ ) machten alle eine Incision in der Cornea zur Besserung des As.

Sie constatirten eine Abflachung des senkrecht zur Incision gerichteten Meridians, welche in der ersten Zeit wach der Operation am grössten ist, nachher langsam abnimmt und schliesslich nicht mehr als $2 \mathrm{D}$ zu betragen scheint. Nach Lucciola entspricht das subjective Ruheund Besserungsgefühl nach der Operation nicht dem relativ kleinen dioptrischen Unterschied vor und nach der Keratotomie.

x) Archives of Ophthalmology. Vol. XXIII. 1894. Siehe Ueber: setzung im Arch. für Augenheilk. XXX. S. 51.

Bates gehört, wie ich einer nachträglichen brieflichen Mittheilung entnehme, unter die folgende Rubrik, der Autoren, welche uicht perforirende Wunden angelegt haben.

2) Nederl, Tijdschr. v. Geneesk. 1895. II. Nr. 11.

3) Arch. f. Augenheilk. XV. S. 178.

4) The surgical Treatment of Astigmatism. Ophthalm. Clinique of Turin. Siehe Uebersetzung in The Ophthalm. Review, Dec. 1896. 
Die Erklärungsversuche des artificiell entstandenen Astigmatismus sind sehr zahlreich.

Viele Autoren suchen die Ursache in der Ausbuchtung der Wunde unter dem Einfluss des intra-ocularen Druckes. Andere suchen die Erklärung in den Widerständen, welche dieser Druck findet. Für alle Einzelheiten verweise ich auf die genannten Arbeiten. Während fast alle Autoren nach dem von Graefe'schen Extractionsschnit eine Abflachung des verticalen Meridians angeben, schreibt Dolganoff1) der Krimmungszunahme in dem Meridian parallel der Incision die wichtigere Rolle zu. Diese letztgenannte Beobaclitung einer Krümmungszunahme des der Schnittrichtung parallelen Meridians, ist sehr interessant und wohl werth, weiter rerfolgt zu werden. Vor allem bedarf der Erklärungsversuch, welcher die Schrumpfung des Narbengewebes als einzige Ursache annimmt, der Bestätigung.

In Bezug auf die Resultate von Pflüger ${ }^{2}$ ), welche ganz von den übrigen abweichen, verweise ich auf die entsprechende Publication und bemerke nur, dass er an myopischen d. h. nicht ganz normalen Augen operirt hat.

\section{Nicht-perforirende Corneawunden.}

Der Eintluss nicht-perforirender Corneawunden wurde bis jetzt wenig studirt und systematische Untersuchungen fehlen noch ganz.

Lucciola ${ }^{3}$ ) publicirt 10 Fälle, in denen er Incisionen gemacht hat, ,ohne den humor aqueus abfliessen zu lassen." Er schliesst rorläufig, dass in diesen Fällen im Gegensatz zur Cataractoperation eine Krümmungszunahme in dem zur Incision parallelen Meridian auftritt. Seine Erklärung, dass auf diese Weise die inneren Theile des

1) Arch, f. Augenheilk. XXIX.

3) La Clinigne Ophtalmologique. Juni 1896.

s) $1 . \mathrm{c}$ 
Experiment. Untersuch, über Entstehung ron Astigmatismus etc. 121

Auges fast unverändert bleiben, und alles auf die Wirkung der Narbe zu beziehen sei, scheint ungenïgend. Pflüger') sah, wenn er nahe dem Cornearand, und diesem parallel, einen Substanzverlust setzte, Krümmungsabnahme in dem Meridian senkrecht zur Incision. Eine Krümmungsänderung in dem der Wunde parallelen Meridian konnte er nicht constatiren, denn: „la guérison se faisait attendre trop longtemps et les patients s'en fatiguaient." Auch diese Publication ist unvollständig.

Straub ${ }^{2}$ ) constatirte eine Abnahme des Astigmatismus in Folge einer Cornealnarbe un $1,5 \mathrm{D}$ bis $0,5 \mathrm{D}$ Maximum vertical.

Ich selbst beobachtete in der Poliklinik Prof. Snellen's eine Patientin, die neben einer Episkleritis einen Astigmatismus von 3,5 D zeigte: im Lauf der Heilung verminderte sich dieser bis auf $1 \mathrm{D}$ Maxim. horizontal. Eine genaue Bestimmung des Astigmatismus vor der Augenkrankheit war nicht gemacht worden; aber mag nun die Anomalie schon vor der Krankheit bestanden haben, oder mag sie, was woll die nächstliegende Anuahme ist, eine Folge der Entziindung gewesen sein, der Fall dürfte wohl beweisen, dass beim Menschen veränderte Spannung am Limbus die Corneakrümmung ändern kann. Wie weit diese Spannungsänderung directe Folge des Entzündungsprocesses in der Sklera ist, wie viel eventuell auf die vermehrte Spannung, respective den Spasmus des nahegelegenen M. rectus inferior, zurïckzuführen ist, wollen wir dahin gestellt sein lassen.

\section{Theoretische Betrachtungen.}

Bevor wir ans Thierexperiment gingen, haben wir versucht, theoretisch die Frage zu lösen, in welcher Weise Entstehung und Heilung von nicht-perforirenden Cornea-

1) 1. $\mathrm{c}$.

马) Nederl. Tijdschr. v. Geneesk. 1892. II. 
wunden die Krümmung der Hornhautoberdäche veränden könnte.

Erstens ist es wahrscheinlich, dass oberflächliche Wunden andere Folgen haben als tiefe. Die Wirkung der ersteren wird gleich nach der Operation null oder mindestens sehr gering sein; erst nachdem sich Bindegewebe gebildet hat, welches sich nach und nach retrahirt, ist die

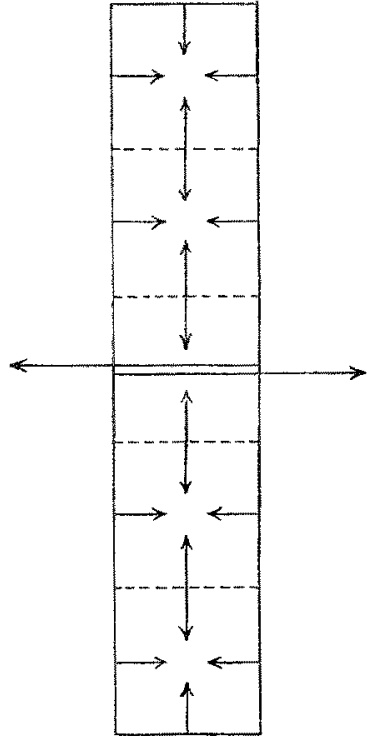

Fig. 1.

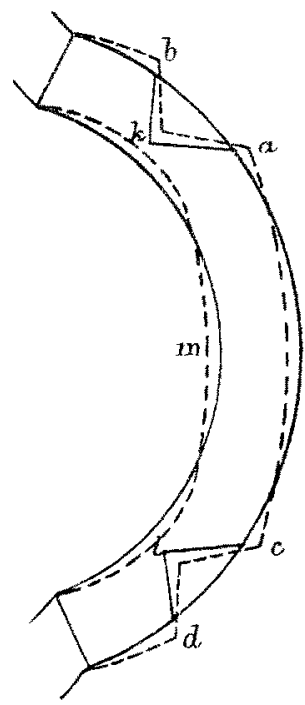

Fig. 2.

Möglichkeit gegeben, dass eine Veränderung der Oberflächenspannung auch eine Veränderung der Corneaober丹läche herbeifuhrt.

A priori ist kaum zu sagen, in welcher Richtung der Narbenzug mit Rücksicht auf die Form der Wunden am meisten wirkt; wahrscheinlich wird nach einer kreisrunden Wunde die Spannung in der Umgebung nach allen Seiten gleichmässig zunehmen. Bei einer ovalen oder mehr länglichen Wunde können wir uns vorstellen, sie bestehe aus einer Anzahl kleinerer Defecte von gleicher Länge und 
Experiment. Untersuch. über Entstehung von Astigmatismus ete. 123

Breite. (Fig. 1.) In Stadium der Narbencontraction wird in jeder dieser Abtheilungen die Oberflächenspannung nach allen Richtungen gleich sein. Während nun die Spannungen, welche in der Längsrichtung der Wunde angreifen, ein grosses Wirkungsgebiet vor sich sehen, sich aber nicht summiren, ist umgekehrt dasjenige der von den Breitseiten wirkenden Kräfte klein, ihre Summe aber viel grösser.

Man möge immer im Ange behalten, dass dies nur der Versuch einer Erklärung ist; alles hängt ab von der Weise, wie der Defect sich schliesst. Ausserdem wird die Richtung der Wunde im Verhältniss zu den Corneameridianen ron grossem Einfluss sein. Es ist also klar, dass wir hier ein sehr complicirtes Problem vor uns haben, dessen theoretische Lösung ich nicht unternehmen will.

Zur Bestimmung der praktischen Werthe dieser Oberfächenspannungen nahm ich die unten erwähnten Versuche an Bällen vor.

Um den Effect tieferer Corneawunden zu beurtheilen, unterscheide ich:

a) directen Einfluss,

b) Einfluss der Narbencontraction.

a) Wenn man an zwei einander gegenüberliegenden Seiten der Cornea ein langes, dem Limbus paralleles, keilförmiges Stück herausschneidet, so werden hierdurch die Spannungsverhältnisse sich ändern und die Cornea wird einem anderen Gleichgewichtsstand in Bezug auf den intraoculären Druck zustreben. Wir setzen voraus, dass dieser in allen Fällen ungefähr constant bleibt.

An der Stelle des Defectes wird der Widerstand abnehmen und in Folge davon eine Ausbuchtung nach vorne entstehen; ihr entsprechend wird dann das Corneacentrum zurücktreten. Schematisch können wir diesen Vorgang abbilden in Fig. 2, wo die ausgezogenen Linien einen meri- 
lionalen Durchschnitt der Cornea vor dem Anbringen der Defecte, und die punktirten Linien den Gleichgewichtszustand nach demselben darstellen.

Man sieht, dass eine Abtlachung der Cornea in den hier gezeichneten Meridian entsteht.

Der Meridian senkrecht zur Ebene der Zeichnung würde durch die centrale Abflachung ebenfalls etwas an Krümmung verlieren, aber auch die peripheren Enden dieses Meridians gehen etwas nach innen (ungefähr in der Richtung uach dem Augenmittelpunkt) in Folge der Vorwölbung nach aussen in dem Parallelkreise da, wo die Defecte liegen; demnach würden, wie man sieht, die verschiedenen Momente einander entgegen wirken und der schliessliche Effect für eine Krümmungsänderung des Meridians, senkrecht zur Zeichnungsebene, dürfte damit selur gering ausfallen.

Als directen Effect keilförmiger, dem Limbus paralleler Wunden, an gegenüberliegenden Seiten der Cornea, kann man eine Krümmungsabnahme in dem Meridian senkrecht zur Wundrichtung erwarten.

b) Einfluss der Xarbencontraetion.

Die Art der Heilung wird gewiss von grossem EinHuss sein. Am wahrscheinlichsten ist es, dass der Corneadefect sich mit Bindegewebe ausfiullt und dass dieses durch seine nachträgliche Schrumpfung versuchen wird, die $W$ undränder einander zu nähern und das besonders in der Richtung senkrecht zum Verlauf des Schnittes. Hierdurch entsteht eine Krümmungszunahme in dem Meridian, welcher direct nach der Operation abgeflacht war. Die Oornea wird versuchen, thre ursprüngliche Form wieder zu erlangen; der aufgetretene As nimmt langsam ab. Es ist nicht wahrscheinlich, dass durch diese Narbencontraction der direct nach der Operation aufgetretene As 
wieder gänzlich verschwinden werde, weil unter dem veränderten Gleichgewichtszustand die Elemente sich dem neuen Zustand angepasst haben und nicht leicht mehr daraus zu bringen sind. Wenn nun aber die Retraction des Narbengewebes immer fortschreitet in der Weise, dass sich die keilförmige Höhle durch das Zusammentreffen der Wundränder $k b$ und $k a, l c$ und $l d$ schliesst, so sollte nach dem Stadium der Krümmungszunahme in dem gezeichneten Meridian, wieder eine Periode von Krümmungsabnahme folgen. Bei aneinander liegenden Wundrändern würde der genannte Meridian sich verkürzt haben, weil etwas Corneagewebe weggefallen ist; der Limbus corneae wird diese Verkürzung hemmen und die Folge dieser auftretenden neuen Spannungsverhältnisse wïrde eine Krümmungsabnahme des gezeichneten Meridians sein.

Theoretisch wïrde hierdurch eine Einbuchtung der Comea entstehen da, wo die Defecte sich befanden (siehe Fig. 3).

Dieses kann aber nur geschehen, wenn das Gewebe der Umgebung sehr resistent ist, da der intraoculare Druck immer dahin trachtet, jeden Theil der Corneaoberfläche

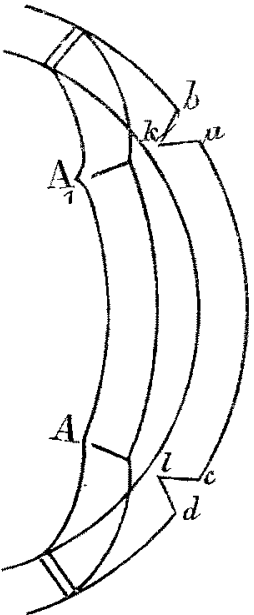

Fig. 3 , in gleiche Krümmung zu versetzen.

Wenn also keine Einbuchtung entsteht, so würde der intraoculare Druck hauptsächlich durch die Spannung der äussersten Cornealamellen getragen, während an der Innenseite unter den geheilten Wunden eine Faltung der Membrana Descemetii und der intact gelassenen Cornealamellen. entstehen würde.

Wie unten exwähnt werden soll, haben oberflächliche, in der Richtung eines Meridians wirkende Spannungen ebenso das Bestreben, diesen abzuflachen; wir können also als wahrscheinlich schliessen: 
Der direct nach der Operation auftetende Astigmatismus wird während der Heilung zuerst abnehmen, vielleicht ungefähr null werden; nachher tritt er wieder in demselben Sinn auf; der Meridian senkrechtzur $W$ undrichtung wird schliesslich abgeflacht sein.

Zur Ergänzung will ich hier noch einige theoretische Betrachtungen mittheilen über Corneadefecte von anderer Lage und Form, wiewohl die Zahl unserer Thierexperimente nach dieser Richtung nicht selrr gross ist. Corneawunden von dieser Art nehmen zu viel von dem centralen Theil der Comea in Anspruch, um praktisch jemals in Betracht zu kommen.

1. An zwei einander gegenüber gelegenen Stellen der Cornea wurden radiäre Wunden gemacht.

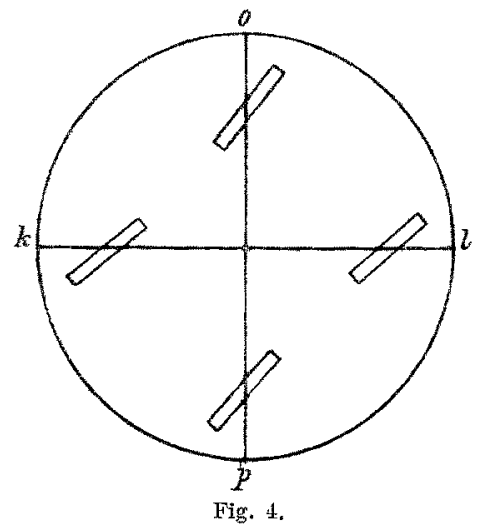

Wahrscheinlieh wird die directe Folge denen der friuher geschilderten Fälle ähnlich sein, aber weniger ansgesprochen, weil die locale Vorwölbung bei geringerer Wundlänge nicht so stark sein wird.

Der Effect der Heilung wird bestehen aus den Folgen einer starken, dem Limbus parallelen Oberflächenspannung an der Stelle der Wunden. Diese strebt, wie die Experimente. mit dem Gummiball erweisen, eine Kritmmungszi-

nahtne des den radiären Wunden parallelen Meridians zu erzeugen; hierdurch wird die direct nach der Operation entstehende Krümmungsabnahme dieses Meridians corrigirt, ja rielleieht übercompensirt.

2. An vier Stellen des Cornearandes, gleich weit von einander entfernt, sind längliche Wunden angebracht, von welchen je zwei mit demselben Meridian einen Winkel von $45^{\circ}$ bilden. (Fig. 4.)

Als directe Folge dieser Defecte wird wahrseheinlich eine centrale Abflachung der Cornea entstehen, weil an der Stelle der 
Wunden, am Limbusende zweier auf einander senkrecht stehenden Meridiane, sie sich etwas vorbuchten wird.

Als unmittelbare Folge der Verwundung wird sich eine Abnahme der Oberflächenspannung ergeben, in dem Meridian senkrecht zur Wundrichtung.

Das entsprechende Experiment mit dem Balle (s. pag. 130) lehrt uns, dass grösssere Spannung in dieser Richtung Krümmungsabnahme in dem den Spannungen parallelen Meridian und Krümmungszunahme in dem hierzu senkrechten Meridian verursacht. Es ist leicht zu begreifen, dass die Entspannung, wie sie direet nach der Operation entsteht, das Umgekehrte herbeiführen wird.

Während der Heilung ist es möglich, dass in Folge der Narbencontraction der direct nach der Operation auftretende Astigmatismus wieder verschwindet, ja selbst, wenn sich die Wundränder einander nähern, in das Gegentheil umschlägt.

\section{Experimente über Oberflächenspannung.}

Wie wir geschildert haben, kann man sich den Einfluss der Heilung auf die definitive Form der Cornea zum Theil abbängig denken von Spannungsrerhältnissen, speciell denen der Corneaoberfläche.

Um eine annähernde Vorstellung zu geben, wie die Kräfte wirken könnten in dem Fall einer Spannung der Oberfläche in bestimmter Richtung, habe ich ein Segment von gleichmässiger Wanddicke aus einem gewöhnlichen Kinderspielball ausgeschnitten. Dureh die Kuppe dieses Segmentes wurde ein Faden gezogen und das erstere daran aufgehängt. Jetzt liess ich das Segment langsam nieder bis die kreisrunde Basis eine weisse Unterlage gerade berührte, ohne dass hierdurch ein nennenswerther Einfluss auf die Form der Basis entstand; wenn ich nun mit einer Nadelspitze ilrer Peripherie folgte, bekam ich eine genaue Projection auf der Unterlage.

Die ausgezogenen Linien in Fig. $\tilde{5}-10$ geben die Form der Basis an im Ruhezustand d. h. bei Ausschluss einer wirkenden Kraft, welche die Basis verändern könnte. Sie stellt einen Kreis dar.

Jetzt brachte ich Spannungen an von verschiedener Grösse und Richtung:

I. Fig. 5. ${ }^{1}$ ) Vermehrte Spannung, einem ganzen Meridian entsprechend.

1) Die Figmren 5 bis 10 sind auf die halbe Grösse reducirt. 
Ein Gummiband $1 \mathrm{~cm}$ breit und $9,5 \mathrm{~cm}$ lang, wurde mit dem einen Ende dureh zwei Häkchen an der Aussenseite des Segments befestigt $\pm 0,5 \mathrm{~cm}$ rom Rand entfernt; danach wurde das Gummiband iuber der convexen Seite ausgespannt in der Richtung eines Meridians und das andere Ende in der gleichen Weise gegenüber befestigt.

Die punktirte Linie zeigt die daraus resultirende Aenderung der Basis; sie hat die Form einer Ellipse angenommen, mit der längeren Achse in der Richtung der Spannung. Die gerade Entfernung zwisehen den beiden Angriffspunkten der wirkenden Kraft hat zugenommen, d. h. die Krümmung in diesem Meridian hat abgenommen oder der Radius ist grösser geworden.

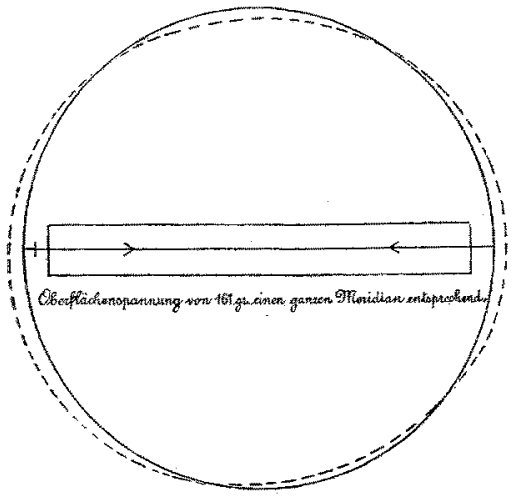

Eig. 5 .

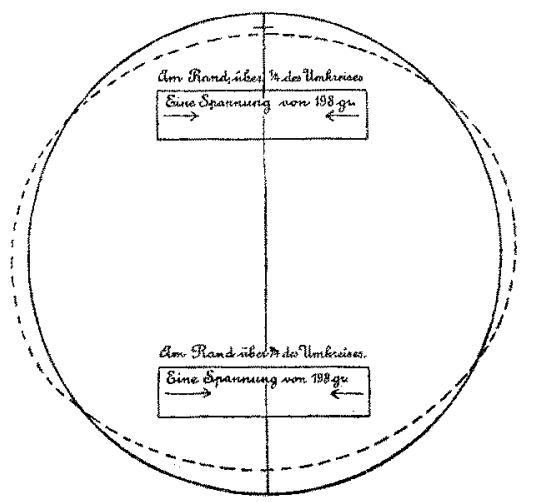

Fig. 6 .

In der Figur ist nieht angegeben, was man beim Versuch deutlich wahrnehmen konnte, dass nämlich unsere Segmentbasis unter dem Einfluss der Streckung des Meridians entsprechend den beiden Endpunkten des letzteren sich etwas von der Unterlage abhob.

Das umgekehrte Verhalten, Krümmung und Länge des Meridians betreffend, muss für den dazu senkrechten Meridian gelten.

Zur Berechnung der Kraft, mit welcher die beiden Enden des Gummibandes einander zustreben, habe ich seine Länge in ansgezogenem Zustand gemessen und danach bestimmte ich das Gewicht, dessen es bedurfte, um die gleiche Länge zu erreichen.

In Fall I war diese Kraft $161 \mathrm{gr}$.

II. Fig. 6. Vermehrte Spannung parallel der Basis über $1 / 4$ des Umisreises. 
Experiment. Untersuch. über Entstehung von Astigmatismus etc. 129

An einer Stelle, $1 \mathrm{~cm}$ vom Basisrand entfernt, wurde ilm parallel eine Oberflächenspannung von $198 \mathrm{gr}$ angebracht über eine Distanz von $1 / 4$ des Umkreises; desgleichen an der gegenüberliegenden Seite.

Dieselbe Betrachtung wie oben lässt uns schliessen, dass die Meridiankrümmung parallel den Spannungen abnehmen wird.

Wie die punktirte Linie in Fig. 6 zeigt, ist auch hier die auftretende Ellipsenform der Basis nicht zu verkennen.

Die Enden des vergrösserten Meridians erheben sich $8,5 \mathrm{~mm}$ und $7 \mathrm{~mm}$ über die Unterlage.

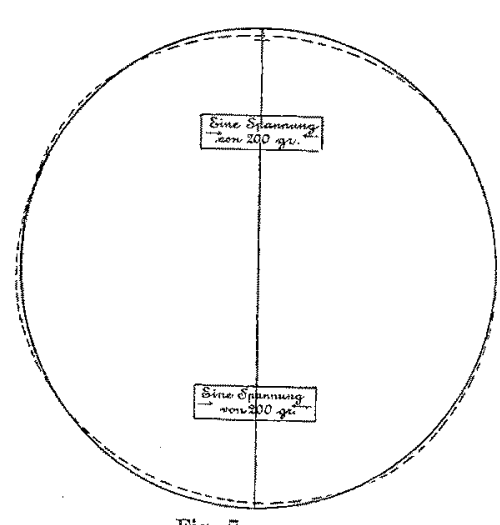

Fig. 7 .

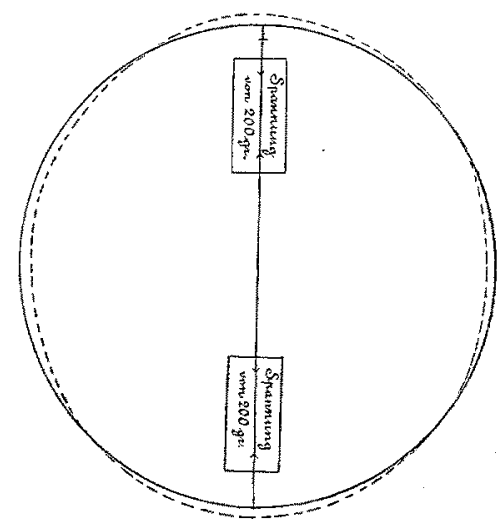

Fig. 8 .

III. Fig. 7. Brachte man eine gleiche Spannung an, in derselben Weise wie bei II, doch auf eine kürzere Strecke, so trat die Aenderung der Basisform auf in demselben Sinn, aber: nicht so stark.

IV. Fig. 8. Hier wirkt an beiden Enden eines Meridians, nicht über die ganze Strecke wie in Fig. 5, sondern nur über einen Theil eine Oberflächenspannung, die die Ellipsenform der Basis zwar nicht so stark wie in Fig. 5, aber doch deutlich und bedeutender als in Fig. 7 auftreten lässt.

V. Fig. 9. Combinirte man III und IV, so musste man mit Beachtung ihrer Resultate erwarten, dass der Effect der Spannung in der Richtung des Meridians grösser sei und, wie Fig. 9 zeigt, ist dies auch so. Zugleich liess sich eine locale Abflachung in der Wirkungssphäre der Kräfte feststellen.

Weiter haben wir noch zwei Kräfte je in einem Winkel von $45^{\circ}$ mit demselben Meridian an dessen beiden Enden angebracht. 
Die Aenderung der Basisform zeigte sich dann ausgesprochen in dem oben gesehilderten Sinn.

VI. Fig. 10. Anf Grund der gewonnenen Resultate dachten wir die Formveränderung am dentlichsten hervor zu bringen, wenn wir vier Spannungen anbrächten, von welchen je zwei einen Winkel von $45^{\circ}$ mit demselben Meridian bildeten, und welche alle, am Rand wirkend, die Formveränderung in demselben Sinn beeinflussen mussten.

Den Effect zeigt Fig. 10.
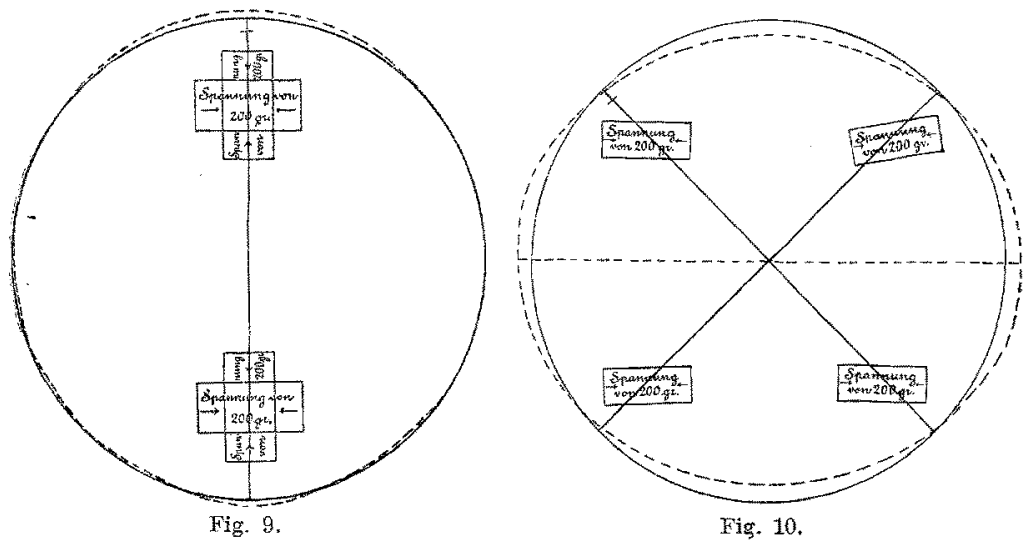

Fig. 10 .

Nachdem das ausgeschnittene Segment wieder in seine alte Lage zum Ball gebracht war mit Hülfe einiger Nähte, versuchte ich, ob vielleicht die oberflächlichen Spannungen, welche die Basisform veränderten, auch die ganze Kugel oder wenigstens jenen Theil der Kngel, welcher an den Rand grenzte, in seiner Form beeinflussten.

Ich war nicht in der Lage, dies sicher zu entscleiden; möglicher Weise waren die Kräfte zu klein. Obwohl an der festen und sehr wenig elastischen Kugel keine direete Veränderung ihrer Form zu constatiren war, so kann man doch annehmen, dass eine während langer Zeit bestehende Spannung die Neigung haben werde, die Form des ganzen Balls in demselben Sinn zu verändern, wie sie das freie Segment verändert.

Ich will nicht behaupten, dass diese Experimente genau darstellen, was geschehen werde bei anomaler Spannung des Corneagewebes und also auch bei Contraction des Narbengewebes: ich meine aber, dass sie uns nützen bei der Erklärung der Entstehung und Richtung des Wundastigmatismus. 
Experiment. Untersuch. über Entstehung von Astigmatismus etc. 131

Beschreibung der Thierexperimente.

Als Versuchsthiere habe ich Kaninchen genommen; zur Bestimmung der Corneakruimmung und des Comearadius diente das Ophthalmometer von Javal-Schiötz.

Die Thiere wurden in der üblichen Weise festgebunden und dabei besonders der Kopf gut fixirt. Es ergab sich nun gleich zu Anfang eine Schwierigkeit dadurch, dass die Thiere beim Versuch das Auge halb oder ganz zukneifen, was die Messung der Cornea mit dem Ophthalmometer natimlich sehr erschwert. Man kann diesem Missstand zwar dureh einen Lidhalter abhelfen, welcher noch eimen kleinen dutten Haken für die Nickhaut trägt; die Schattenseiten dieser Methode aber sind Austrocknung der Cornea oder auch stärkerer Thränenfluss, da die 'Thiere gewöhnlich heftig mit den Lidern pressen.

Im Verlauf des Versuchs ergaben sich noch weitere Schwierigkeiten. Wenn man, nachdem das Thier festgebunden und der Kopf genau horizontal gestellt ist, das Ophthalmometer scharf einstellte, so komte man bemerken, dass einzelne, direct einander folgende Beobachtungen rerschiedene Resultate gaben.

Es wurde mir bald klar, dass diese durch Bewegungen des Kaninchenauges verursacht wurden, wodurch man die Corneakrümmong jedesmal an einer anderen Stelle bestimmte.

Un solche Drehungen auszuschliessen, rersuchte ich eine Stelle auf der Comea zn markiren, nm immer auf sie einznstellen. Damit ein solcher Punkt im Ophthalmometer zur Wahinelimung gelangt, was bei Anwendung von Tusche nicht der Fall ist, erwies es sich mir am zweckmässigsten, nach Anaesthesirung mit Cocain, die Cornea 10-11 Secunden lang mit einem Krystall von Bleiacetat zu touchiren, wodureh eine punktförmige Bleincrustation entsteht, welche Monate lang als ein kleiner hellweisser Fleck sichtbar bleibt.

Bei jeder Beobachtung wurle nun das Auge des Kaninchens so vor dem Ophthalmometer aufgestellt, dass die Doppelbilder des Pünktchens symmetrisch standen, d. l. gleichweit entfernt waren von den Aussenrändern der beiden mittleren Figuren (siehe Fig. 11). Wenn man die Corneakrümmung in einem anderen Meridian bestimmt, so drehen sich die Pünktehen mit und behalten ilhe symmetrische Lage.

Ich suchte immer die Pünktchen genau in der Mitte der Cornea anzubringen, weil damit die Anfstellung des Thieres vor 
dem Ophthalmoneter vereinfacht war. Liegt der Punkt aber etwas excentriscl, so kann man dem Fehler abhelfen, indem man den Kopf entsprechend dreht, um dadureh die geforderte Lage des Punktes zu den mittleren Figuren zu erreichen.

Wenn man immer gut darauf achtete, so war man sicher, die Krümmung immer an derselben Stelle zu bestimmen. Uebrigens ist es nach eimiger Uebung nicht sehwer, genau das Centrum zu finden.

Ausserdem war ich immer darauf bedacht, Thierkopf und Tubus genau horizontal zu stellen, damit auch eventuelle Rollbewegungen direet nachweisbar wären.

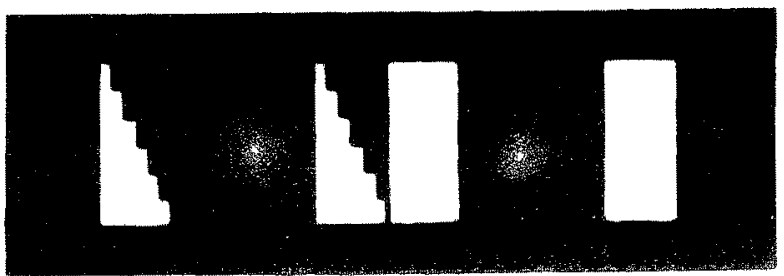

Fig. 11.

Cm festzustellen, ob etwa liese geschilderten Vorbereitungen irgendwie die Corneavbertläche alteriren, andererseits $o b$ im Lauf melnerex lionate sich an den Corneaverhältnissen etwas ändert, brachte ich an zwei Angen nur solche Punkte an, und liess eines ganz intact.

Ich betrachtete die Thiere als geeignet für die weiteren Experimente, wenn nach der gesehilderten Vorbereitung mindestens drei Untersuchungen wăhrend einiger Tage das gleiche Resultat ergaben.

Nicht seiten muss man nach dem Anbringen der eigentlichen Wunden, deren Ziel die Erzeugung des Astigmatismus ist, mehrere Tage warten, ehe man untersuchen kann, da Reizerscheinungen auftreten, die eine genaue Bestimmung verhindern.

Ich benntzte 3 Methoden, um nieltperforirende Corneawunden zu erzeugen: 1) Schneiden, 2) A uskratzen, 3) Brennen.

\section{Sehnittwunden.}

Bei dieser Form der Verwundung liegt die Gefalır nahe, die Cornea ganz zn durchtrenmen.

Bei allen genannten Methoden, besonders beim Brennen, 
Experiment. Untersuch. über Entstehung von Astigmatismus etc. 133

wulsten sich die Wundränder; dadureh scheint die Wunde tiefer als sie wirklich ist.

An 3 Versuchsthieren machte ieh nichtperforirende Selnittwunden:

\section{Verlauf der Experimente.}

Mit einem v. Graefe'schen Messer machte ich $2 \mathrm{~mm}$ nach innen rom Limbus corneae, diesem parallel, oben und unten, jedesmal über $1 / 4$ des Corneaumkreises, keilförmige Wunden.

Kaninchen, I. O. S.

Projection des den Wunden ungefähr parallelen Meridians $+70^{\circ}$ temporal.

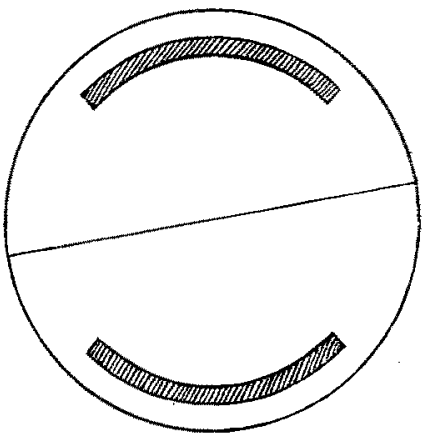

Tig. 12.

\begin{tabular}{|c|c|c|c|c|}
\hline & As. & $\begin{array}{l}\text { Bichtung des } \\
\text { Meridians stärk- } \\
\text { ster Krïmmung }\end{array}$ & $\begin{array}{l}\text { Cornearadius } \\
\text { in dem Meri- } \\
\text { dian den Inci- } \\
\text { sionen parallel }\end{array}$ & $\begin{array}{l}\text { Cornearadius } \\
\text { in dem Meri- } \\
\text { dian zu den } \\
\text { Incisionen } \\
\text { senkrecht }\end{array}$ \\
\hline & & & $\mathrm{mm}$ & $\mathrm{mm}$ \\
\hline $\begin{array}{l}\text { Vor der Operation } \\
\text { 16. Januar Operation }\end{array}$ & kein As. & temporal & 6,77 & 6,77 \\
\hline $26 . \quad, \quad 4 \quad$ & $1,5 \mathrm{D}$ & $70^{\circ}$ & $6,46^{\circ}$ & 6,64 \\
\hline 29. & $1,5 \mathrm{D}$ & $70^{\circ}$ & 6,58 & 6,77 \\
\hline 30. & $1,5 \mathrm{D}$ & $65^{\circ}$ & 6,58 & 6,77 \\
\hline 11. Febuar & $1,5 \mathrm{D}$ & $70^{\circ}$ & 6,58 & 6,77 \\
\hline 20 & $1 D$ & $70^{\circ}$ & 6,70 & 6,83 \\
\hline 1. Mäzz & $1 \mathrm{D}$ & $60^{\circ}$ & 6,77 & 6,89 \\
\hline $6 . \quad \%$ & $1 \mathrm{D}$ & $60^{\circ}$ & 6,77 & 6,89 \\
\hline 9. & $1 \mathrm{D}$ & $50^{\circ}$ & 6,77 & 6,89 \\
\hline 11. & $1 D$ & $70^{\circ}$ & 6,77 & 6,89 \\
\hline 17. & $I D$ & $70^{\circ}$ & 6,77 & 6,89 \\
\hline 22. & $\frac{1}{1} \mathrm{D}$ & $70^{\circ}$ & 6,77 & 6,89 \\
\hline 15. Mai & D & $65^{\circ}$ & 7,10 & 7,24 \\
\hline 16., & $1 \mathrm{D}$ & $65^{\circ}$ & 7,10 & 7,24 \\
\hline
\end{tabular}




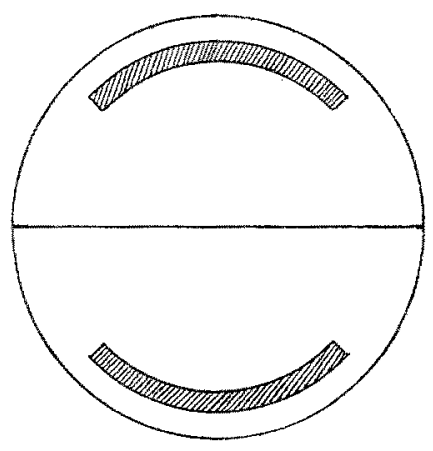

Kaninchen II. O. D.

Projection des den Wanden ungefähr parallelen Veridians horizontal.

Fig. 13.

\begin{tabular}{|c|c|c|c|c|}
\hline & As. & $\begin{array}{l}\text { Richtung des } \\
\text { Meridians } \\
\text { stärkster } \\
\text { Krummung. }\end{array}$ & $\begin{array}{l}\text { Cornearadius in } \\
\text { dem Meridian } \\
\text { den Incisionen } \\
\text { parallel. }\end{array}$ & $\begin{array}{l}\text { Cornearadius in } \\
\text { dem. Meridiun zu } \\
\text { den Incisionen } \\
\text { zenkreeht. }\end{array}$ \\
\hline & & & $\min$ & $\mathrm{mm}$ \\
\hline $\begin{array}{l}\text { Vor der Operation } \\
20 \text {. Febr. Operation }\end{array}$ & kein As. & & 6,58 & 6,58 \\
\hline 2. $"$ & $1,5 \div 2 \mathrm{D}$ & horizontal & $6, \breve{02}$ & 6,70 \\
\hline 27. & 1 à $1,5 \mathrm{D}$ &, & 6,46 & 6,64 \\
\hline 4. März & $\quad 0,5 \mathrm{D}$ & , & 6,46 & 6,64 \\
\hline 9. & $0,5 \mathrm{D}$ & , & 6,83 & 6,89 \\
\hline 18. & $0,5 \mathrm{D}$ & , & 6,77 & 6,89 \\
\hline 21., & $0,5 \mathrm{D}$ & , & 6,96 & 7,03 \\
\hline
\end{tabular}

Resultat: Nach nichtperforirenden Schnittwunden, dem Limbus corneae parallel, entsteht direct ein Astigmatismus von 1,5 bis $2 \mathrm{D}$; der Meridian stärkster Krümmung verläuft den Incisionen parallel. - Bei Kaninchen II entsteht dieser Astigmatismus durch Zunahme des Radius in dem senkrechten Meridian; nach 25 Tagen war hier eine stärkere Abnahme des Radius in fem den Incisionen parallelen Meridian zu constatiren. Bei Versuchsthier I und III entsteht der Astigmatismus direct nach der Operation durch Krümmungszunahme des den Incisionen parallelen Meridians. Der dazu senkrechte Meridian nimmt an Krümmung weniger zu (Fall $I$ ) oder behält seine ursprüngliche Gestalt (Fall III). Während der Wundheilung vermindert sieh der Astigmatismus langsam: in 2 Fallen (I und II) blieb er ungefähr noch einen Monat constant, in Fall III vergrösserte der Astigmatismus sielı um $0,5 \mathrm{D}$. 
Experiment. Untersuch. über Entstehung von Astigmatismus etc. 135

Kaninehen III. O. D.

Projection des Meridians, ungefähr den Wunden parallel, $+40^{\circ}$ temporal.

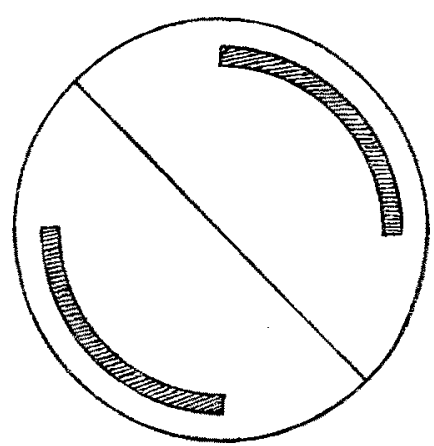

Fig. 14.

\begin{tabular}{|c|c|c|c|c|}
\hline & As. & $\begin{array}{l}\text { Richtung } \\
\text { des Meridians } \\
\text { stärkster } \\
\text { Krümmung. }\end{array}$ & $\begin{array}{l}\text { Cornearadins } \\
\text { in dem } \\
\text { Meridian } \\
\text { den Incisionen } \\
\text { parallel. }\end{array}$ & $\begin{array}{l}\text { Cornearadius } \\
\text { in dem } \\
\text { Meridian zu } \\
\text { den Ineisionen } \\
\text { senkrecht. }\end{array}$ \\
\hline $\begin{array}{l}\text { Vor der Operation } \\
\text { 18.Februar Operation }\end{array}$ & kein As. & temporal & $\begin{array}{l}\mathrm{mma} \\
6,96\end{array}$ & $\begin{array}{r}\mathrm{mm} \\
6,96\end{array}$ \\
\hline $\begin{array}{l}20 . \\
24 .\end{array}$ & $\begin{array}{l}1,5 \mathrm{D} \\
1 \mathrm{D}\end{array}$ & $\begin{array}{l}40^{\circ} \\
45^{\circ}\end{array}$ & $\begin{array}{l}6,77 \\
6,83\end{array}$ & 6,96 \\
\hline 2. März & $1 \mathrm{D}$ & $45^{\circ}$ & $\begin{array}{l}0,80 \\
6,83\end{array}$ & $\begin{array}{l}\text { 0,96 } \\
6,96\end{array}$ \\
\hline 9. & $1 \mathrm{D}$ & $40^{\circ}$ & 6,83 & 6,96 \\
\hline 12. " & $1,5 \mathrm{D}$ & $40^{\circ}$ & 6,77 & 7,03 \\
\hline 18. " & $1,5 \mathrm{D}$ & $45^{\circ}$ & 6,77 & 7,03 \\
\hline 21. " " & $1,5 \mathrm{D}$ & $40^{\circ}$ & 6,77 & 7,03 \\
\hline
\end{tabular}

II. Kratzwunden.

Verlauf der Experimente.

Hier wurde mit einem Messer mit Sicherheitsvorrichtung, welehes ein zu tiefes Eindringen verhütet, auf der Cornea vorgezeichnet, in welcher Richtung man auskratzen wollte; danach bediente ich mich eines speciell dazu verfertigten Löffelchens und kratzte so viel Corneagewebe aus, dass in deutlicher Defect entstand von ungefähr $0,3 \mathrm{~mm}$ Tiefe.

a) $2 \mathrm{~mm}$ nach innen rom Limbus, diesem parallel, oben und unten, jedesmal über $1 / 4$ des Corneaumkreises, wurde Corneagewebe ausgekratzt. 
Kaninchen IV. O. S. Projection des Meridians, ungefähr den Wunden parallel horizontal. (S. Fig. 15.)

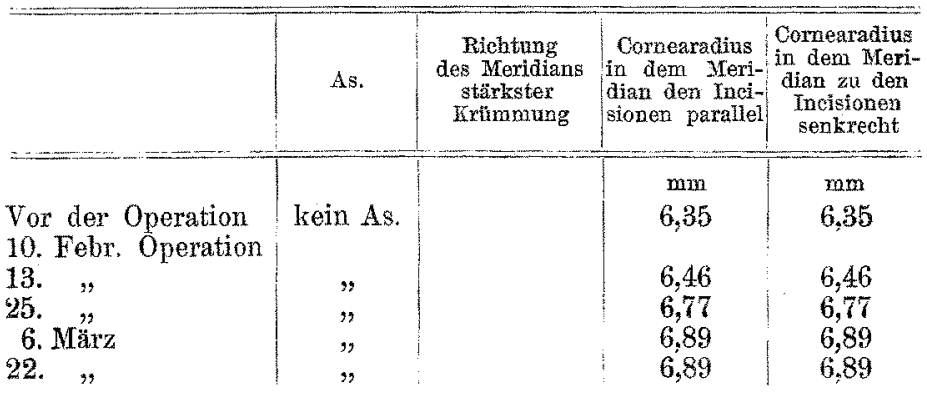

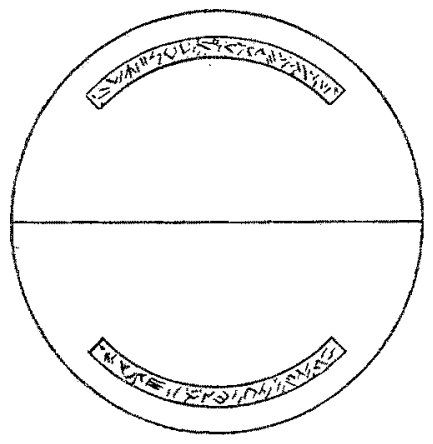

Fig. 15 .

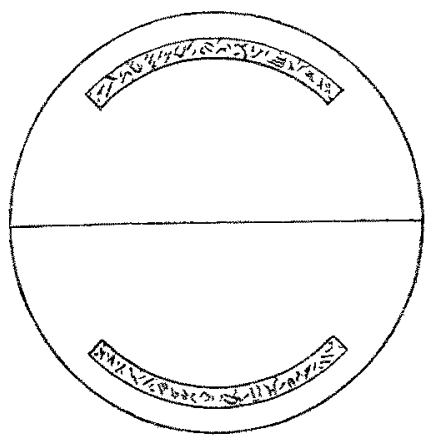

Fig. 16.

Kaninchen V. O.S. Operation und Wundheilung wie bei IV. (S. Fig. 16.)

\begin{tabular}{|c|c|c|c|c|}
\hline & As. & $\begin{array}{l}\text { Dichtung } \\
\text { des Meridians } \\
\text { stärkster } \\
\text { Krümmoung }\end{array}$ & $\begin{array}{l}\text { Cornearadius } \\
\text { in dem Meri- } \\
\text { dian den Inci- } \\
\text { sionen parallel }\end{array}$ & $\begin{array}{l}\text { Comearadius } \\
\text { in dem Meri- } \\
\text { dian zu den } \\
\text { Incisionen } \\
\text { senkrecht }\end{array}$ \\
\hline $\begin{array}{l}\text { Vor der Operation } \\
\text { 12. Febr. Operation } \\
\text { 4. März } \\
\text { 12. " } \\
\text { 16. " } \\
\text { 18. " } \\
23 . ",\end{array}$ & $\begin{array}{c}\text { kein As. } \\
1 \mathrm{D} \\
\text { kein As. } \\
" \\
" \\
" \\
"\end{array}$ & horizontal & $\begin{array}{l}6,89 \\
6,89 \\
6,96 \\
7,08 \\
7,03\end{array}$ & $\begin{array}{l}\operatorname{man} \\
6,52 \\
7,03 \\
6,89 \\
6,96 \\
7,03 \\
7,03\end{array}$ \\
\hline
\end{tabular}


Experiment. Untersuch. über Entstehung von Astigmatismus etc. 137

Kaninehen VI.

b) Oben und unten $2 \mathrm{~mm}$ nach innen vom Limbus, wurden Defeete ausgekratzt $+3 \mathrm{~mm}$ lang und breit.

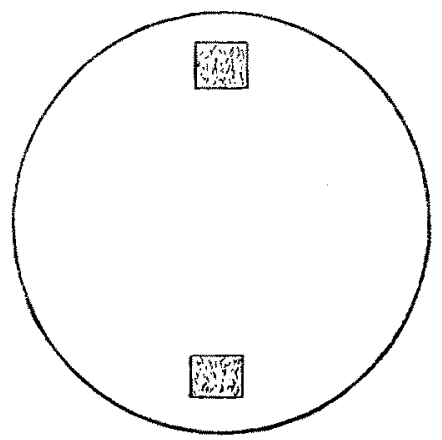

Fig. 17 .

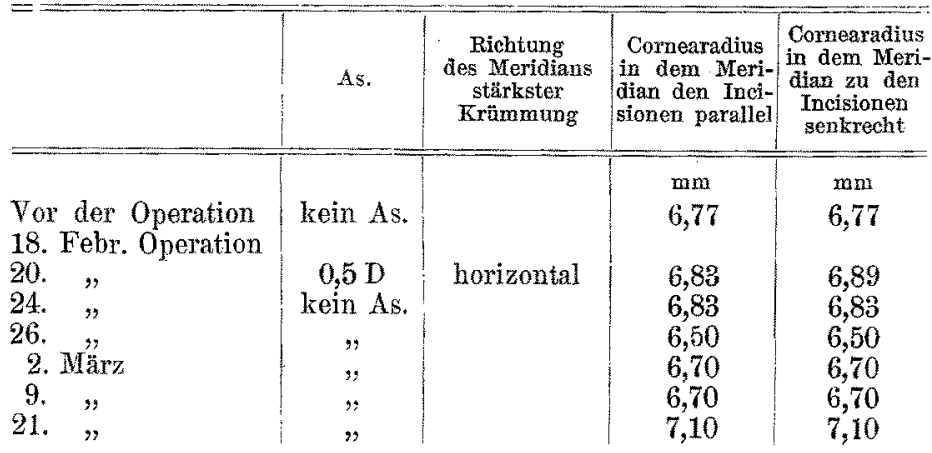

Resultat. Nichtperforirende Kratzwunden verursachen direct nach der Operation einen Astigmatismus von 0,5 bis $1 \mathrm{D}$ durch Krümmungsabnahme in dem senkreehten Meridian ${ }^{2}$ ). Später geht der Astigmatismus ganz zurück.

Der Cornearadins vermehrt sich zuerst am meisten in dem senkrechten Meridian; die letzten Bestimmungen zeigten gleichmässige Radiuszunahme in allen Meridianen.

\section{Brandwunden.}

Verlauf der Experimente.

Mit dem Galvanocauter brannte ich Wunden von versehiedener Tiefe, Richtung und Grösse.

a) $2 \mathrm{~mm}$ nach innen rom Limubus, diesem parallel, oben und unten, jedesmal über ${ }^{1 / 4}$ des Corneaumkreises wurden Defeete gebrannt.

1) Im Folgenden ist unter "senkrecht" und ,parallel" immer zu verstehen: senkrecht zur Wundrichtung und parallel zur Wundrichtung. 
Kaninchen VII. O.S. Wunden oberflächlich, Projection des den Wunden ungefähr parallelen Meridians horizontal. (S. Fig. 18.)

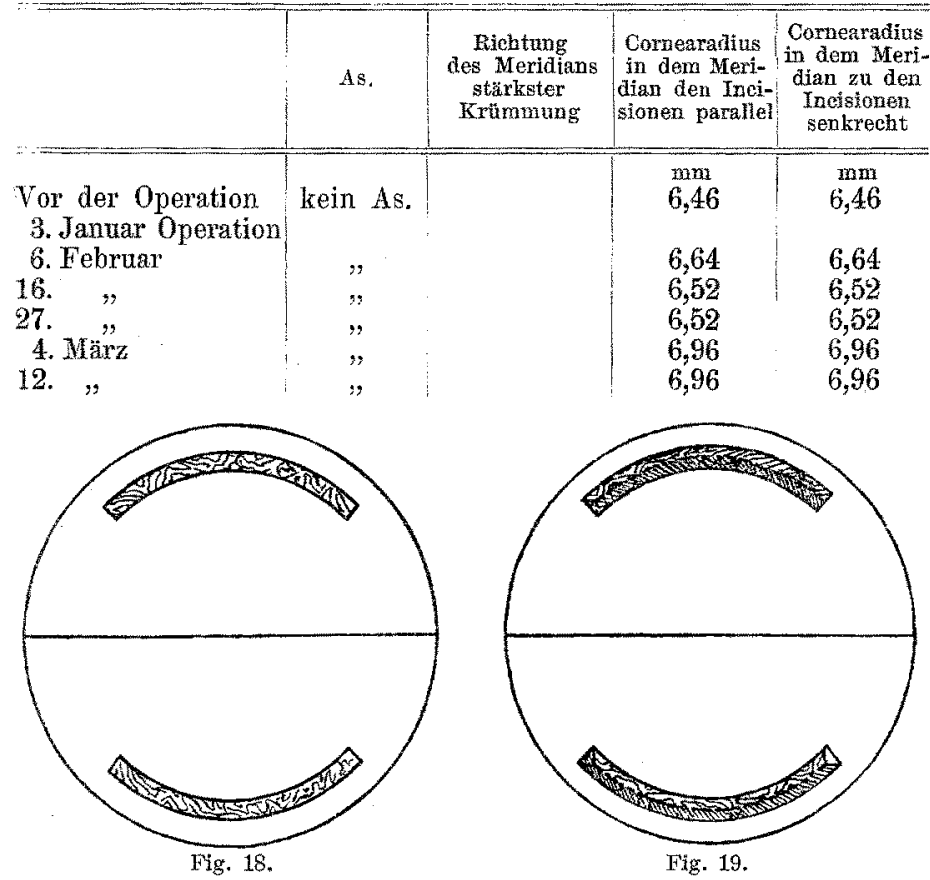

Kaninchen VIII. O.D. Richtung der Wunden wie bei a). Wunden tief. (S. Fig. 19.)

\begin{tabular}{|c|c|c|c|c|}
\hline & As. & $\begin{array}{l}\text { Richitung } \\
\text { des Meridians } \\
\text { stärkster } \\
\text { Krǘmmung }\end{array}$ & $\begin{array}{l}\text { Comearadius } \\
\text { in dem Meri- } \\
\text { dian den Ind- } \\
\text { sionen parallei }\end{array}$ & $\begin{array}{l}\text { Comearadius } \\
\text { in den Meri- } \\
\text { dian zu den } \\
\text { Incisionen } \\
\text { senkreeht }\end{array}$ \\
\hline $\begin{array}{l}\text { Vor der Operation } \\
\text { 2. Febr. Operation }\end{array}$ & kein As. & & $\begin{array}{l}\mathrm{mm} \\
6,64\end{array}$ & $\operatorname{mim}_{6,64}$ \\
\hline $5 . \quad, \quad, \quad$ ( & $4 \mathrm{D}$ & $80^{\circ}$ nasal & & $?$ \\
\hline & $3 \mathrm{D}$ & $75^{\circ} \quad$, & 6,30 & 6,52 \\
\hline 11. & $2,5 \mathrm{D}$ & $80^{\circ}$ & 6,35 & 6,58 \\
\hline 13. " & $3 \mathrm{D}$ & $80_{0} \quad$, & 6.80 & 6,52 \\
\hline 25. & $6 \mathrm{D}$ & horizontal & $?$ & $?$ \\
\hline 2. Mâxz & $6 \mathrm{D}$ & $80^{\circ}$ nasal & $6,64 ?$ & $7,47 ?$ \\
\hline & $6 \mathrm{D}$ & horizontal & 6,41 & 7,10 \\
\hline & $6 \mathrm{I}$ & ", & 6,41 & 7,10 \\
\hline & $6 \mathrm{D}$ & & 6,41 & 7,10 \\
\hline & $6 \mathrm{D}$ & $80^{\circ}$ nasal & 6,46 & 7,17 \\
\hline
\end{tabular}


Experiment. Untersuch. über Entstehung von Astigmatismus etc. 139

Kaninchen IX. O. D.

Operation wie bei a). Wunden tief. Projection des den Wunden ungefähr parallelen Meridians $20^{\circ}$ temporal.

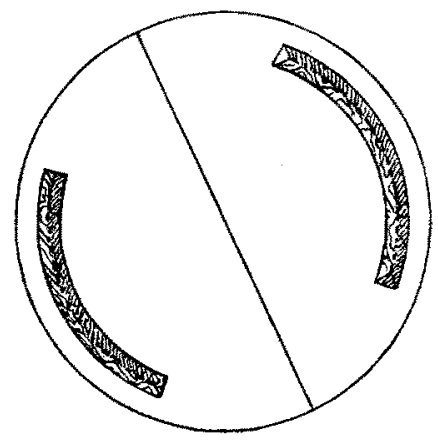

Fig. 20.

\begin{tabular}{|c|c|c|c|c|}
\hline & As. & $\begin{array}{l}\text { Richtung } \\
\text { des Meridians } \\
\text { stä́rkster } \\
\text { Krümmung }\end{array}$ & $\begin{array}{l}\text { Cornearadius } \\
\text { in dem Meri- } \\
\text { dian den Inci- } \\
\text { sionen parallel }\end{array}$ & $\begin{array}{l}\text { Cornearadius } \\
\text { in dem Meri- } \\
\text { dian zu den } \\
\text { Incisionen } \\
\text { senkrecht }\end{array}$ \\
\hline $\begin{array}{l}\text { Vor der Operation } \\
\text { 3. Febr. Operation }\end{array}$ & kein As. & & $\begin{array}{l}\mathrm{mm} \\
6,64\end{array}$ & $\begin{array}{l}m m \\
6,64\end{array}$ \\
\hline 13. " & $6 \mathrm{D}$ & Vertical & 6,64 & 7,39 \\
\hline 16. " & $6 \mathrm{D}$ & $"$ & 6,64 & 7,39 \\
\hline 24. & $6 \mathrm{D}$ & & 6,30 & 6,96 \\
\hline 2. März & $5 \mathrm{D}$ & $20^{\circ}$ temp. & 6,30 & 6,83 \\
\hline 6. & $4 \mathrm{D}$ & Vertical & 6,52 & 6,96 \\
\hline 11. & $3 \mathrm{D}$ & 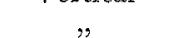 & 6,64 & 6,96 \\
\hline 18. " & $3 \mathrm{D}$ & 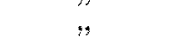 & 6,58 & 6,89 \\
\hline 21. , & $3 \mathrm{D}$ & $"$ & 6,58 & 6,89 \\
\hline 23. & $3 \mathrm{D}$ & & 6,35 & 6,64 \\
\hline 16. Mai & $3 \mathrm{D}$ & $10^{\circ}$ temp. & 6,70 & 7,17 \\
\hline
\end{tabular}

Resultat: Nach oberfächlichen Brandwunden entsteht kein Astigmatismus. Die Corneakrümmung nimmt in allen Meridianen gleichmässig ab.

Als directe Folge länglicher; dem Limbus paralleler, tiefex Brandwunden kann man einen Astigmatismus von 4 bis $6 \mathrm{D}$ constatiren, wobei der Meridian stärkster Krümmung den Incisionen parallel verläuft.

Während der Wundheilung vermindert sich dieser Astigmatismus bedeutend.

In Fall VIII vergrössert er sich nachher in demselben Sinn, in Fall IX bleibt er nach einem Monat constant.

Der Cornearadius in dem den Wunden parallelen Meridian nimmt $a b$ in Fall VIII direct nach der Operation, doeh ver- 
längert er sich später wieder langsam; daneben kann man eine geringere Abnahme des Cornearadius in dem senkreehten Meridian constatiren, aber nachher überwiegt die Zunalime, sodass er schliesslich der bedentend längere ist.

In Fall IX bleibt direct nach der Operation der Cornearadius in dem den Wunden parallelen Meridian gleichgross and der auftretende Astigmatismus entsteht nur durch' Zunahme des Radius in dem senkrechten Meridian. Dieser nimmt später, im ersten Monat nach der Operation, gleichmässig $a b$, während der erstgenannte Radius nach einigen Schwankungen viel geringere Abnahme zeigt.

Nach drei Monaten ist die Radiuszunahme nicht für alle Meridiane gleichmässig, sondern in dem zu den Wunden senkrechten Meridian am grössten.

b) Die Beschreibung der folgenden Experimente sei hier kurz angegeben.

Nasal und temporal werden radiäre Wunden gebrannt.

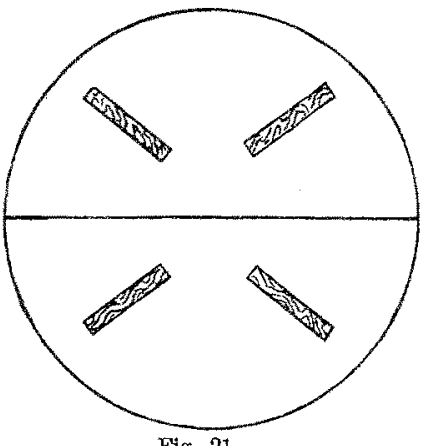

Kaninehen $\mathrm{X} . \mathrm{O}$. S.

Projection des den Wunden nngefähr parallelen Meridians horizontal.

Fig. 21 .

\begin{tabular}{|c|c|c|c|c|}
\hline & As. & $\begin{array}{l}\text { Richtung } \\
\text { des Meridians } \\
\text { stürkster } \\
\text { Krïmmung }\end{array}$ & $\begin{array}{c}\text { Cornearadius } \\
\text { in dem } \\
\text { Meridian den } \\
\text { Incisionen } \\
\text { parallel }\end{array}$ & $\begin{array}{l}\text { Cornearadius } \\
\text { in dem Meri- } \\
\text { dian za den } \\
\text { Incisionen } \\
\text { senkrecht }\end{array}$ \\
\hline & & & $\mathrm{mm}$ & $\mathrm{mm}$ \\
\hline $\begin{array}{l}\text { Vor der Operation } \\
\text { 4. März Operation }\end{array}$ & kein As. & & 6,77 & 6,77 \\
\hline 16. $", \quad+$ & $5 \mathrm{D}$ & $70^{\circ}$ temporal & 6,41 & 6,64 \\
\hline 19. & $2 \mathrm{D}$ & $80^{\circ}$ & 6,64 & 6,89 \\
\hline & 1,50 & horizontal & 6,70 & 6,77 \\
\hline 23. & 1,50 & $70^{\circ}$ nasal & 6,52 & 6,70 \\
\hline 15. Mai & $1 \mathrm{D}$ & $70^{\circ} \quad$, & 7,10 & 7,24 \\
\hline 16. & D & $70^{\circ}$ & 7,17 & 7,32 \\
\hline
\end{tabular}


Experiment. Untersuch. über Entstehung von Astigmatismus etc. 141

K a ninchen XI, O, D. Wunden oberflächlich. Projection des den Wunden ungefahr parallelen Meridians horizontal (s. Fig. 22).

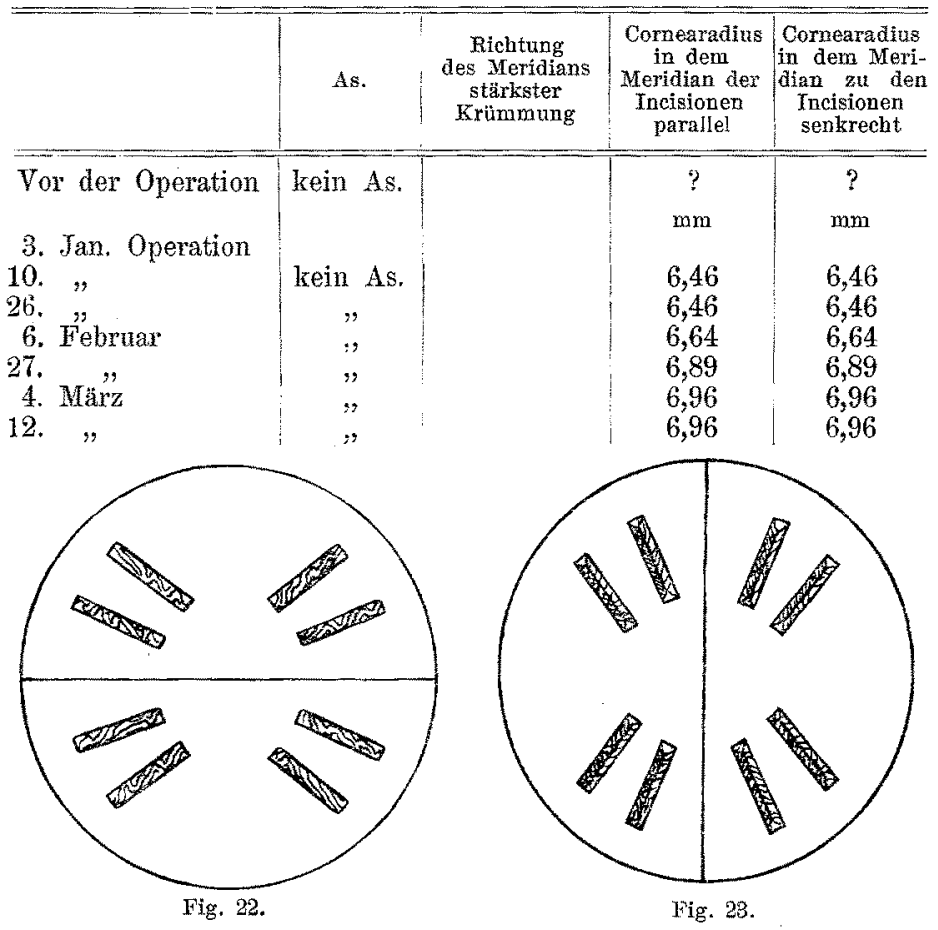

Kaninchen XII. O.D. 4 radiäre Wunden sind hier oben und unten gebrannt worden: die Defecte sind von mässiger Tiefe. Projection des den Wunden ungefähr parallelen Meridians vertical (s. Fig.23).

\begin{tabular}{|c|c|c|c|c|}
\hline & As. & $\begin{array}{c}\text { Richtung } \\
\text { des Meridians } \\
\text { stärkster } \\
\text { Krümmung }\end{array}$ & $\begin{array}{l}\text { Cornearadius } \\
\text { in dem } \\
\text { Meridan den } \\
\text { Incisionen } \\
\text { parallel }\end{array}$ & $\begin{array}{l}\text { Cornearadins } \\
\text { in dem Meri- } \\
\text { dian an den } \\
\text { Incisionen } \\
\text { senkrecht }\end{array}$ \\
\hline $\begin{array}{l}\text { Vor der Operation } \\
\text { 4. Jan. Operation. }\end{array}$ & Kein As. & & $\begin{array}{l}\mathrm{mm} \\
6,64\end{array}$ & $\begin{array}{l}\min \\
6,64\end{array}$ \\
\hline 28. "Februar & $\begin{array}{ll}1,5 & \mathrm{D} \\
1 & \mathrm{D}\end{array}$ & $\begin{array}{c}\text { vertical } \\
20^{\circ} \text { temporal }\end{array}$ & $\begin{array}{c}6,46 \\
?\end{array}$ & $\begin{array}{c}6,64 \\
?\end{array}$ \\
\hline $20 . \quad$, & $1,5 \mathrm{D}$ & $20^{\circ}$ temporal & 6,70 & 6,89 \\
\hline 1. März & & vertical & 6,77 & 6,89 \\
\hline $9 . \quad \%$ & $1 \mathrm{D}$ & vertical & 6,96 & 7,10 \\
\hline 16. & $1 \mathrm{D}$ & $20^{\circ}$ temporal & 7,03 & 7,17 \\
\hline & $1 \mathrm{D}$ & vertical & 7,03 & 7,17 \\
\hline
\end{tabular}




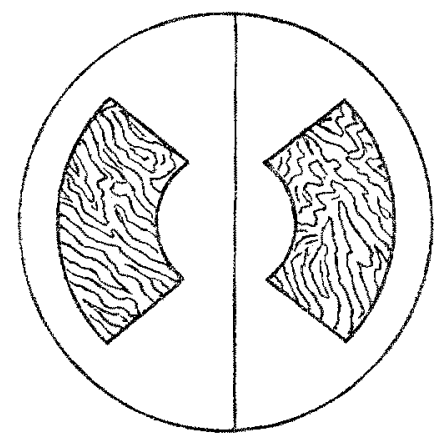

Fig. 24 .

\section{Kaninchen XIII. O. S.}

Sectorenförmige Wunden sind an der nasalen und temporalen Seite gebrannt. Die Wunden sind nicht tief. Projection des den Wunden parallelen Meridians vertical.:

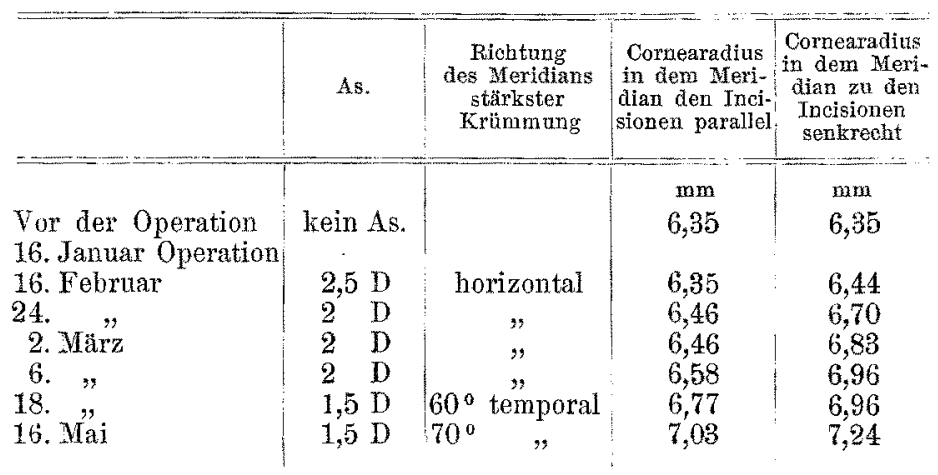

Resultat: Nach kleinen radiären, oberflächlichen Wunden entsteht kein Astigmatismus. Die Radien der Cornea verlängern sich in allen Meridianen gleichmässig.

Nach radiären, tiefen Wunden ist direet (nach 12 Tagen) Astigmatismus zu constatiren; der Meridian stärkster Krümmung verläuft den Wunden parallel. Dieser Astigmatismus nimmt sofort $a b$ und bleibt später constant.

Der Cornearadius in dem den Wunden parallelen Meridian verkürzt sich in Fall $\mathrm{X}$ und $\mathrm{XII}$. Die Zunahme in dem hierzu. senkrechten Meridian ist in Fall XIII und XIV besonders klar.

Schliesslich ist bei allen Experimenten die Zunahme des Radius in dem senkrechten Meridian grösser als in dem parallelen Meridian. 
Experiment. Untersuch, über Entstehung von Astigmatismus ete. 143

Die sectorenförmigen Wunden, wie in Fall XIII kann man sich denken als zusammengesetzt aus mehreren radiären Streifen. Der direct nach der Operation entstehende Einfluss war hier nicht zu bestimmen. Die überwiegende Zunahme des Radius in dem zu den Wunden senkrechten Meridian ist hier nicht zu verkennen.

Die Form der Wunden in Fall XIV stellt einen Uebergang dar von radiären zu grösseren sectorenähnlichen Defecten. D́as Resultat ist das gleiche wie bei den anderen Experimenten.

Mit Bezug auf das Seite 130 erwähnte Ballexperiment mag lier noch die Besehreibung eines Thierversuches folgen. (S. Fig. 26.)

Kaninchen XIV. O. S.

Nasal und temporal sind zwei mässig tiefe Defecte $\pm 3 \mathrm{~mm}$ lang und $+2 \mathrm{~mm}$ breit gebrannt. Projection des den Wunden ungefähr parallelen Meridians $60^{\circ}$ temporal.

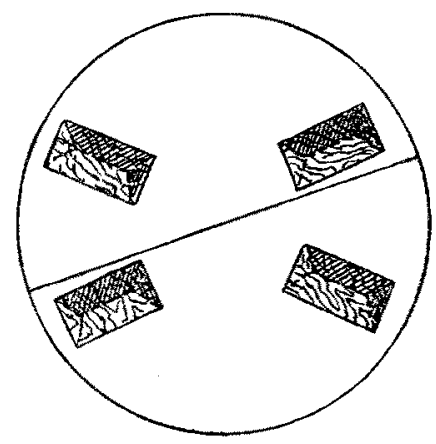

Fig. 25

\begin{tabular}{|c|c|c|c|c|}
\hline & As. & $\begin{array}{c}\text { Richtung } \\
\text { des Meridians } \\
\text { stänster } \\
\text { Krümmung }\end{array}$ & $\begin{array}{l}\text { Cornearadius } \\
\text { in dem Mexi- } \\
\text { dian den Inci- } \\
\text { sionen parallel }\end{array}$ & $\begin{array}{l}\text { Cornearadius } \\
\text { in dem Meri- } \\
\text { dian zu den } \\
\text { Incisionen } \\
\text { senkrecht }\end{array}$ \\
\hline $\begin{array}{l}\text { Vor der Operation } \\
\text { 16. Januar Operation }\end{array}$ & kein As. & & $\begin{array}{l}\mathrm{mm} \\
6,35\end{array}$ & $\begin{array}{l}\mathrm{mm} \\
6,35\end{array}$ \\
\hline 16. Februar & $2,5 \mathrm{D}$ & $65^{\circ}$ temporal & 6,41 & 6,70 \\
\hline & $2 \mathrm{D}$ & $60^{\circ} \quad$, & 6,52 & 6,77 \\
\hline 2. März & $3 \quad \mathrm{D}$ & $60^{\circ}$ & 6,41 & 6,77 \\
\hline $6 . \quad$, & $\mathrm{D}$ & $65^{\circ}$ & 6,41 & 6,77 \\
\hline 11. & $3 \quad \mathrm{D}$ & $65^{\circ}$ & 6,41 & 6,77 \\
\hline 18. & $3 \quad \mathrm{D}$ & $60^{\circ}$ & 6,52 & 6,89 \\
\hline 15. Mai & $2,5 \mathrm{D}$ & $60^{\circ}$ & 6,96 & 7,32 \\
\hline 16. , & $2,5 \mathrm{D}$ & $65^{\circ}$ & 6,96 & 7,32 \\
\hline
\end{tabular}




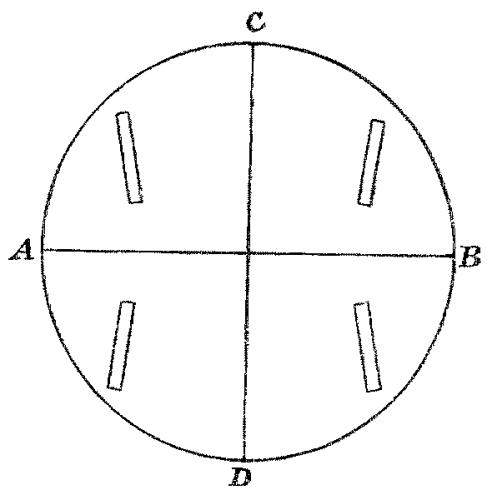

Kaninchen XV. (O. D.

Vier Brandwunden, von welchen je zwei einen Winkel von $45^{\circ}$ mit demselben Meridian bilden, sind hier am Comearand angebracht.

Fig. 26.

\begin{tabular}{|c|c|c|c|c|}
\hline & A.s. & $\begin{array}{c}\text { Richtungy } \\
\text { des Meridians } \\
\text { stiarkster } \\
\text { Krümmung }\end{array}$ & $\begin{array}{l}\text { Cornearadius } \\
\text { in dem } \\
\text { Meridian AB }\end{array}$ & $\begin{array}{c}\text { Cornearadins } \\
\text { in dem } \\
\text { Meridian } \\
\text { CD }\end{array}$ \\
\hline $\begin{array}{l}\text { Vor der Operation. } \\
\text { 4. März Operation }\end{array}$ & Kein As. & & $\begin{array}{c}\mathrm{mm} \\
6,77\end{array}$ & $\begin{array}{r}\mathrm{mm} \\
6,77\end{array}$ \\
\hline 16. & $\pm \tilde{5}$ & $70^{\circ}$ temporal & 6,46 & 7,03 \\
\hline 17. & $\mathrm{D}$ & $70^{\circ}$ temporal & 6,35 & 6,89 \\
\hline 18. , , & $\mathrm{D}$ & horizontal & 6,46 & 6,77 \\
\hline 19. , & $3 \mathrm{D}$ & horizontal & 6,58 & 6,89 \\
\hline 15. Mai & $1,5 \mathrm{D}$ & $80^{\circ}$ temporal & 7,10 & 7,32 \\
\hline
\end{tabular}

Resultat: Direct nach der Operation entsteht ein Astigmatismus, in dem Sinn, dass Meridian $\mathrm{AB}$ sich stärker krümmt. Nach zwei Monaten vermindert sich dieser Astigmatismus allmählig. Der Comearadius im Meridian $\mathrm{AB}$ zeigt zuerst $\mathrm{Ab}$ nahme, später Zunahme: diese Zunahme ist nicht so gross wie in dem Meridian CD, welcher schon direct nach der Operation schwächere Krüminung angab.

\section{Controlversuche.}

Das Corneacentrum ist nit einem Krystall von Bleiacetat tonehirt. 
Experiment. Untersuch. über Entstehung ron Astigmatismus etc. 145

Kaninchen XVI. 0. S.

\begin{tabular}{l|c|c}
\hline \hline & As.' & Cornearadius \\
\hline \hline & kein As. & mm \\
31. December &, & 7,01 \\
12. Januar & $"$ & 7,03 \\
7. Februar & $"$ & 7,03 \\
25. " & $"$ & 6,89 \\
4. März & $"$ & 6,96 \\
16. " & $"$ & 7,17 \\
22. " & $"$ & 7,55 \\
15. Maì & &
\end{tabular}

Kaninchen XVII. O. S.

\begin{tabular}{l|c|c}
\hline \hline & As, & Cornearadius \\
\hline & & mm \\
9. Januar & kein As. & 6,70 \\
10. ", & $"$ & 6,70 \\
12. " & $"$ & 6,70 \\
13. " & $"$ & 6,70 \\
20. " & $"$ & 6,70 \\
25. Februar & $"$ & 7,03 \\
4. März & $"$ & 6,96 \\
11. " & $"$ & 6,96 \\
23. " & $"$ & 7,10 \\
15. Mai & $"$ & 7,47
\end{tabular}

Kaninchen XVIII. O. D.

\begin{tabular}{c|c|c}
\hline \hline & As. & Cornearadius \\
\hline & & mm \\
9. Januaz & kein As. & 6,70 \\
10. " & 1/2 D V. & $?$ \\
12. " & kein As. & 6,64 \\
13. " &, & 6,70 \\
20. " & $"$ & 6,70 \\
25. Februar & $"$ & 6,83 \\
4. März & $"$ & 6,77 \\
11. " & $"$ & $?$ \\
23. " & $"$ & 6,83 \\
15. Mai & $"$ & 7,63
\end{tabular}

จ, Graefe's Archiv für Ophthalmologie. XLV. 
Kaninchen XIX. O. D. Dieses Versuchsthier blieb ganz intact: Man war hier nicht ganz sicher, die Krümmung immer in demselben Meridian zu bestimmen.

\begin{tabular}{|c|c|c|c|c|}
\hline & As, & $\begin{array}{l}\text { Richtung } \\
\text { dess Mleridians } \\
\text { stärrster } \\
\text { Krutummung }\end{array}$ & $\begin{array}{c}\text { Cornearadius } \\
\text { in dem } \\
\text { Meridian } \\
\text { stärkster } \\
\text { Krümmitug }\end{array}$ & $\begin{array}{l}\text { Cornearadius } \\
\text { in dem } \\
\text { Meridian } \\
\text { schwhehster } \\
\text { Krônmmung }\end{array}$ \\
\hline & & & $\operatorname{mm}$ & $\mathrm{mm}$ \\
\hline 3. Januar & $1 \mathrm{D}$ & $40^{\circ}$ temporal & 6,77 & 6,89 \\
\hline $26 . \quad 3$ & $1,5 \mathrm{D}$ & $20^{\circ}$ & $?$ & $?$ \\
\hline 7. Eebruar & $1 \mathrm{D}$ & rertical & 6,89 & 6,96 \\
\hline 25. & $1, \hat{b} \mathrm{D}$ & $\%$ & 6,89 & 7,10 \\
\hline 4. März & kein As. & 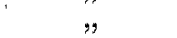 & 7,08 & 7,03 \\
\hline 16., & $1 \mathrm{D}$ & 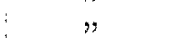 & 7,03 & 7,17 \\
\hline 22. & $2 \mathrm{D}$ & 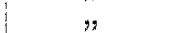 & 6,90 & 7,24 \\
\hline 15. Mai & $1 D$ & , & 7,24 & 7,39 \\
\hline
\end{tabular}

Resultat: Bei im Corneacentrum touchirten Versuchsthieren tritt nach $4 \frac{1}{2}$ bis 5 Monaten kein Astigmatismus auf. Also entsteht weder durch diese Beliandlung noch durch das Wachsthum Astigmatismus. Greichmässige allmählige Radiuszunahme ist bei allen Versuchsthieren $z$ u constatiren.

In Fall XIX muss man die Schlüsse mit grosser Vorsicht ziehen, da man meistens gar nicht wusste, in welchem Meridian unan die Krümmung bestimmte; daher erklären sich die grossen Differenzen, welche man bei verschiedenen Messungen fand.

Eine allmählige Zunalme des Radius in allen Meridianen lässt sich nicht verkennen.

Indem ich die Beschreibung lieser Versuche abschlesse, will iel noch kurz den Heilungsverlaut der Wmaten und die pathologiseh-anatomische Untersuehning besprechen. Trotz den angewendeten antiseptischen Methoden trat nicht selten einen 'Tag' nach der Operation eitrige Conjunctivitis anf; ibrigens bewhite sich die Reinigung der Conjunetiva mit $3 \%$ Borsäurelösung gut. Besonders bei Brandwunden konnte man selbst nach zeln Tagen noch die verdickten Wundränder sehen. Sohnitt- und Kratzwunden heilten viel schneller. Nach einem Monat war von der Wunde selbst meistens nichts mehr zu sehen, ausgenommen eine grauweisse, nachher sich aufhellende stelle.

Oberfächliche Wunden zeigten in iluem Narbengewebe dem blossen Auge oft keine Differenz gegen normales Corneagewebe.

Nach kleinen Wunden schien mir das Narbengewebe sich rascher aufzuhellen. 
Experiment. Untersuch, über Entstehung von Astigmatismus etc. 147

Tabelle zum Vergleich einiger Differenzen der Cornearadien bei den Controlversuchen und bei Versuchsthiel" I bis IX (wo also ungefahr gleiche Wunden gesetzt sind) zwischen dem Zeitpunkt vor der Operation und:

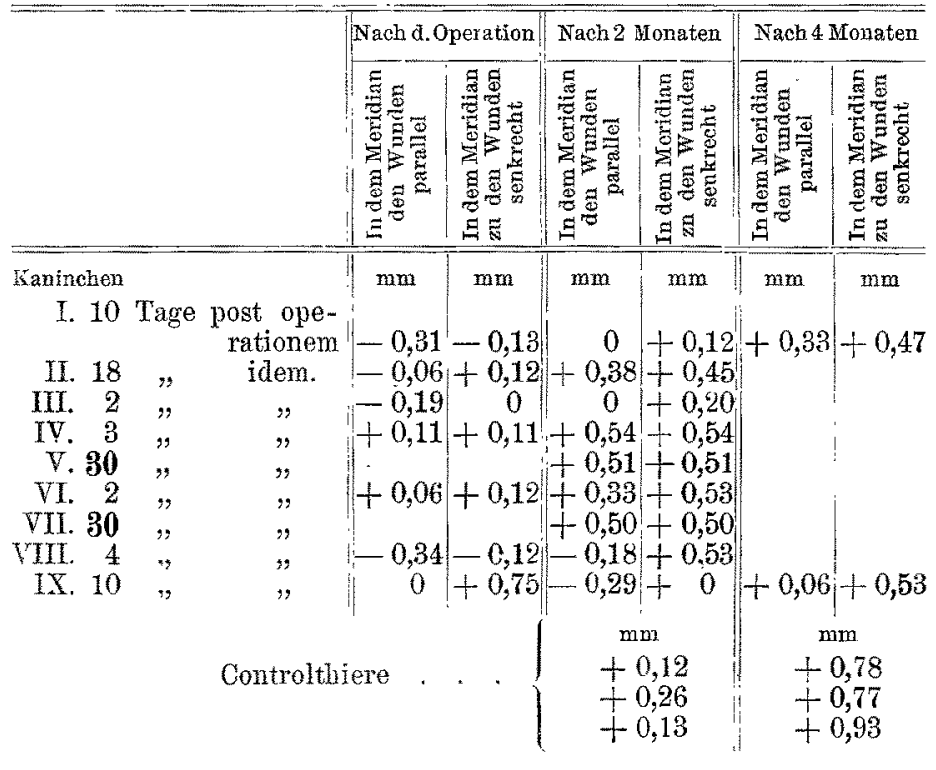

Resultat: In drei Füllen (I, III, VIII) ist direet nach der Operation hauptsächlich $A b n a h m e$ des Radins in dem paralleleu Meridian zu constatiren; in drei Fällen (II, VI, IX) gehört die Zunahme des Radius besonders dem senkrechten Meridian an. In einen Fall (IV) ist diese Zunahme in beiden Meridianen gleich.

Wir können also im Voraus nicht bestimmen, wieviel von einem gleich nach der Operation auftretenden Astigmatismus auf Krümmungszunahme des parallelen, oder auf eine Krümmungsabnahme des senkrechten Meridian kommt.

Im Allgemeinen ist nach zwei Monaten die Zunahme des Radius bei den Versuchsthieren grösser als bei den Controlthieren; nach vier Monaten ist bei den letzteren diese Zunahme überwiegend. negativ.

Die pathologisch-anatomische Untersuchung war zum Theil

Die Augen wurden in Celloidin eingebettet und mit Haematoxylin und Eosin gefärlut. An den Augen mit oberfächlichen 
Wunden konnte man die Narbe oft schwer finden, an jenen mit tiefen Defecten sah man als Beweis des ganz geheilten Processes eine dem normalen Corneagewebe fast ganz gleiche Substanz, nur schienen die Corneafasern in der Narbe etwas unregelmässig gelagert.

Von einer Faltung der Membrana Descemetii oder der unter der Wunde liegenden Corneaelemente war absolut nichts zu sehen.

Auch am Limbus war alles normal; die Tiefe der Wunden schien ungefähr $2 / 3$ der Corneadicke zu betragen.

\section{Ueborsicht der Resultate.}

Wenn wir die gewomnenen Resultate uibersehen, so können wir schliessen, dass durch nichtperforirende, dem Limbus parallele Corneawunden an beiden Enden desselben Meridians direct ein Astigmatismus entsteht in demselben Sinn wie theoretisch angenommen wurde, d. h. also: die Achse stärkster Krümmung liegt parallel der Wundrichtung.

Die Experimente zeigen uns aber, dass direct nach der Operation die Krümmungszunahme des der Wunde parallelen Meridians von grösserem Einfluss ist, als wir anf S. 124 meinten. In der einen Hälfte der Fälle wurde nämlich ein derartiges Verhalten constatirt, während in der anderen Hälfte eine Abflachung des zu der Wunde senkrechten Meridians festgestellt werden konnte. Diese Zunahme der Krimmung in dem Meridian parallel zur Wundrichtung bei Versuchsthier I, III, VIII muss wahrscheinlich so erklärt werden, dass die Wunden zu ansgedehnt waren, wodurch die corneo-sklerale Verbindung über eine zu grosse Strecke zu schwach wurde und also die Hornhaut im Ganzen dem intraocularen Druck nachgeben, konnte. Daraus folgte dann eine Verkürzung sämmtlicher Radien und unter diesen Verhältnissen kann die Zunahme des Radius, welche in dem zu den Wunden senkrechten Meridian theoretisch erwartet wurde, theils oder ganz aufgehoben, ja sogar übercorrigirt werden. 
Experiment. Untersuch. über Entstehung von Astigmatismus etc. 149

Constant ist jedenfalls das Verhalten, dass die stärkste Krümmung in dem $z u$ den Wunden parallelen Meridian gefunden wird.

Aus unseren Resultaten geht weiter hervor, dass während der Heilung der Astigmatismus sich vermindert, dass also die Vorbuchtung der Wunde abnimmt; weiter ergiebt sich aber, dass keine Faltung der Cornea auftritt, und dass, wie theoretisch möglich erschien, nicht in allen Fällen der nachträgliche Narbenzug Zunahme des Astigmatismus verursacht. Nur Fall VIII giebt direct nach der Operation $4 \mathrm{D}$ Astigmatismus, welcher zuerst langsam zurïck geht und nach 23 Tagen, um welche Zeit der Narbenzug sich sehr bemerklich macht, sich wieder vermehrt um $3 \mathrm{D}$; dieses stimmt genau mit unseren theoretischen Betrachtungen überein.

Die Auskratzung des Corneagewebes ist für unseren Zweck keine gute Methode, bessere Resultate giebt das Brennen. Man kann sich dies auch wohl erklären, inden man annimmt, dass die Kratzwunden zu oberflächlich waren, während beim Brennen anch die weitere Umgebung der verletzten Stelle mit alterirt wird; durch das letztere Moment mag die locale Ausdehnung der Cornea gefördert werden.

Das mikroskopische Bild zeigt, dass die Membrana, Descemetii und die intact gelassenen Cornealamellen unter der $W$ unde unverändert geblieben sind. Auch diese Thatsache stiitzt die Behauptung, dass der Narbenzug den senkrechten Meridian nicht viel verkürzen kann.

Die Resultate weisen darauf hin, dass nur Wunden, deren Tiefe ungefähr ${ }^{2} / 3$ der Corneadicke beträgt, Astigmatismus hervorrufen können.

Die Resultate, die unsere Vorversuche am Gummiball gegeben haben, sind auf oberflächliche Narben der Cornea nicht direct zu übertragen; denn bei ihr ist die als Folge der Wunde auftretende Spannungsänderung praktisch ohne Belang. 
Auch auf die Dauer kam diese Spanmungsänderung die Cornea- und Skleraelemente in ihrer gegenseitigen Lage nicht ändern.

Die lange nach der Operation beobachteten Fälle lassen schliessen, dass der Astigmatismus nach einiger Zeit constant bleibt: die Cornea ist' in ihrer Form bleibend geändert.

Bemerkenswerth ist übrigens, dass auch der nach perforirenden Wunden auftretende Astigmatismus, dem hier Erörterten gleichsinnig ist.

Die Resultate der anderen Versuche stimmen nur zum Theil mit den theoretischen Betrachtungen uberein.

Die Beobachtungen von Fall XV deuten auf Entspannung der Cornealamellen, wodurch der zu den Wunden senkrechte Meridian an stärksten gekrümmt wird. Auch hier kann der Narbenzug den direct auftretenden Astigmatismus nicht ganz vernichten und entgegengesetzten Astigmatismus entstehen lassen.

Der Vorgang bei den radiären Wunden lässt sich schwer erklären: ihre Resultate stimmen zwar mit den theoretischen Annahmen gut überein, aber ihre Entwicklungsformen weichen wesentlich von der postulirten ab.

Bald nach der Operation tritt ein Astigmatismus auf in dem Sinn, dass der den Wunden parallele Meridian am stärksten gekrümmt wird; diese Krümmungsveränderung ist aber wahrscheinlich nicht die erwartete, durch starke dem Limbus parallele Oberfächenspannung verursachte, da sie unter der Wundheilung sich vermindert, während man vielmehr Zunahme des Astigmatismus erwarten musste in Folge der sich summirenden, in jeder Wunde gleichgerichteten Narbencontraction.

Wie man hier den direct auftretenden Astigmatismus erklären muss, kann ich nicht entscheiden. Ich war nicht in der Lage, weitere Untersuchungen zur Lösung dieser Frage zu machen. Uebrigens scheint mir diese Methode auch praktisch nicht gat branchbar zu sein. 
Experiment. Untersuch. über Entstehung von Astigmatismus etc. 101

Nothwendig ist es, auch die Controlthiere genau zu beobachten. Sie beleuchten unsere Versuche nach rerschiedenen Richtungen und beweisen:

1. dass nicht etwa mit dem Wachsen der Thiere Astigmatismus entsteht.

2: dass bei Kaninchen ron einem Alter, wie sie zu Versuchen meistens benutzt werden, der Cornearadius nach und nach grösser wird, die Oberfäche also an Krümmung abnimmt. Daraus mag sich auch erklären, dass bei den Versuchsthieren gleichfalls der Radius nach einigen Monaten in allen Meridianen zunahm. Hier ist aber die Zunahme nicht so bedentend wie bei den Controlthieren, was darauf hinweist, dass die Operation und ihre Folgen die normale Entwicklung der Cornea etwas gehemmt haben.

Wir haben hiermit zwei neue Resultate, welche vielleicht für andere Untersuchungen von Werth sein können.

Nach Mittheilung meiner Resultate sei es mir gestattet, kurz meine Meinung zusammen zu fassen iiber die praktischen Ergebnisse dieser Methode in der Frage der Erzengung eines Astigmatismus, respective der operativen Correction eines vorbandenen.

Legen wir mittelst des Galvanocauters zwei nichtperforirende Corneawunden an, welche, $2 \mathrm{~mm}$ nach innen yom Limbus, diesem parallel verlaufen an zwei entgegengesetzten Seiten eines Meridians und welche circa 1/4 des Umkreises einnehmen, während ihre Tiefe circa $\% / 3$ der Corneadicke beträgt, so sind wir im Stande, einen bleibenden Astigmatismus von 3 bis $6 \mathrm{D}$ hervorzurufen in dem Sinn, dass die Achse der stärksten Krümmung der Wundrichtung parallel, also senkrecht zu dem obengenannten Meridian verläuft; dabei kann sowohl die Länge des Radius in dem Meridian parallel zur Wundrichtung abnehmen, als der Radius des hierzu senkrechten Meridians zunehmen, d. h. es kamm myopischer oder hyper- 
152 L. J. Lans, Exp. Untersuch. üb. Entstehung v. Asti̊gmatismus etc. metropischer Astigmatismus entstehen. Wenden wir unsere Methode bei bestehendem hypermetropischen oder myopischen Astigmatismus an, so kann also die Refraction nach der Heilung sowohl Emmetropie als Myopie oder Hyperopie sein. Nach unseren Erfahrungen wird wahrscheinlich durch längere Wunden hypermetropischer Astigmatismus, durch kürzere, breitere Wunden myopischer Astigmatismus geheilt.

Ich bin nun weit davon entfernt, zu glauben, dass etwa mit diesen Versuchen die Frage der Correction des Astigmatismus erledigt sei. Vielmehr muss ich gestehen, dass die Frage der Dosirung noch ganz offen ist. Ich hoffe aber, eine Richtung gezeigt und späteren Untersuchungen damit vorgearbeitet zu haben.

Die gefundene Methode scheint mir noch nicht sicher genug, um in Fällen von hochgradigem Astigmatismus welcher durch Cylindergläser noch corrigirt werden kann, die operative Behandlung zu empfehlen, aber für die Eälle von complicirtem, unregelmässigem Astigmatismus, welche jeder bisherigen Behandlung spotten, scheint mir ein Versuch in der hier angewiesenen Richtung berechtigt zu sein.

Zum Schlusse ist es mir eine angenehme Pflicht, Herrn Prof. Dr. Koster für seine Unterstiitzung bei Ausfuhrung der Arbeit meinen herzlichen Dank auszusprechen. 